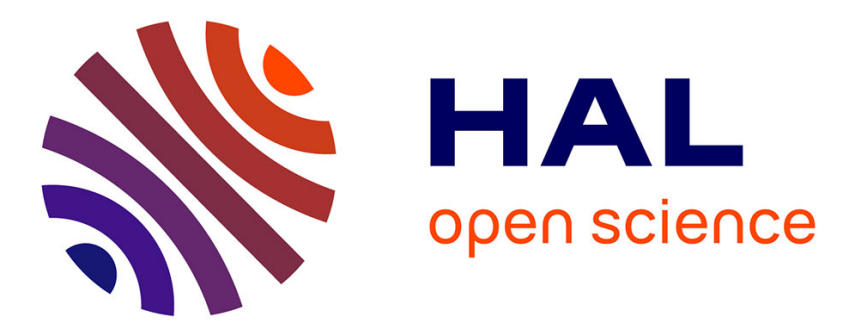

\title{
Conséquences d'un principe d'extremum en turbulence
}

B. Castaing

\section{To cite this version:}

B. Castaing. Conséquences d'un principe d'extremum en turbulence. Journal de Physique, 1989, 50

(2), pp.147-156. 10.1051/jphys:01989005002014700 . jpa-00210909

\section{HAL Id: jpa-00210909 https://hal.science/jpa-00210909}

Submitted on 1 Jan 1989

HAL is a multi-disciplinary open access archive for the deposit and dissemination of scientific research documents, whether they are published or not. The documents may come from teaching and research institutions in France or abroad, or from public or private research centers.
L'archive ouverte pluridisciplinaire HAL, est destinée au dépôt et à la diffusion de documents scientifiques de niveau recherche, publiés ou non, émanant des établissements d'enseignement et de recherche français ou étrangers, des laboratoires publics ou privés. 
Classification

Physics Abstracts

$47.10-03.40 \mathrm{G}$

\title{
Conséquences d'un principe d'extremum en turbulence
}

\author{
B. Castaing
}

Centre de Recherches sur les Très Basses Températures, C.N.R.S., BP 166X, 38042 Grenoble Cedex, France

(Reçu le 28 avril 1988, révisé le 30 août, accepté le 26 septembre 1988)

Résumé. - De nombreux auteurs ont proposé que l'écoulement turbulent observé soit celui qui rend extremale telle ou telle quantité. Ces principes d'extremum n'ont jamais pu être généralisés. Nous proposons dans cet article de postuler l'existence d'une telle quantité sans l'expliciter et de tirer les conséquences de cette simple existence. Sur l'exemple très souvent étudié de la turbulence développée ces conséquences semblent en accord avec ce que l'on connaît ou admet : distribution $\log$ normale du flux d'énergie $\varepsilon$ de Kolmogorov, loi en $\exp -\left(\frac{k}{k_{0}}\right)^{\beta}$ pour $\varepsilon$ fonction du vecteur d'onde $k$. Dans notre formalisme de plus, $\beta$ apparait comme la codimension des structures dissipatives et nous prédisons que $\beta=\beta_{0} / \log R_{\mathrm{e}}$, où $\beta_{0}$ est une constante et $R_{\mathrm{e}}$ le nombre de Reynolds, à la limite où $R_{\mathrm{e}} \rightarrow \infty$.

Abstract. - Many authors have proposed that the extremum of some quantity determines the observed turbulent flow. None of these quantities have allowed a generalization of the corresponding extremum principle. We propose here to examine the consequences of the only existence of such a quantity, without explicitation. Taking as an example the often studied developped turbulence, we show that these consequences are in agreement with what is known or accepted about it: $\log$ normal distribution for the Kolmogorov energy flux $\varepsilon$, stretched exponential law $\exp -\left(\frac{k}{k_{0}}\right)^{\beta}$ for $\varepsilon$ as a function of the wave vector $k$. In such a formalism moreover, $\beta$ appears as the co-dimension of the dissipative structures. We predict that in the high Reynolds number $\left(R_{\mathrm{e}}\right)$ limit, $\beta=\frac{\beta_{0}}{\log R_{\mathrm{e}}}$ where $\beta_{0}$ is a constant.

\section{Introduction.}

Dans cet article, nous discutons l'utilité d'un principe d'extremum en turbulence développée. C'est un sujet sensible. La première tentative en ce sens est attribuée à Von Karman [1]. De nombreux auteurs s'y sont ensuite essayé, certains parmi les plus prestigieux. Les approches les plus voisines de ce que nous allons essayer de faire sont celles de R. Benzi et al. [2] et de T. M. Brown [3]. Mais à notre connaissance - limitée tant la littérature est abondante sur ce sujet -, dans toutes ces tentatives la quantité dont il fallait chercher l'extremum a été 
précisément désignée. Nous proposons ici d'y renoncer tout d'abord et de chercher quelles conséquences pourrait avoir la simple existence d'une telle quantité.

Cela ne veut pas dire que nous n'avons aucune idée de ce que doit être cette quantité. Cela signifie plutôt que nous ne savons pas la relier a priori aux grandeurs mesurables de l'écoulement. Les considérations qui suivent dans cette introduction n'ont donc aucune prétention de rigueur. Elles ne sont pas des définitions. Elles sont là pour expliquer pourquoi nous attendons de la quantité en question les propriétés que nous lui prêterons. Nous ne présenterons le formalisme lui-même que dans le paragraphe 3 .

Il est communément admis [4] que le nombre de degrés de liberté d'un écoulement croît vite avec son nombre de Reynolds $R_{\mathrm{e}}$, comme $R_{\mathrm{e}}^{9 / 4}$. Ce nombre de degrés de liberté devient donc bien supérieur au nombre de grandeurs nécessaires pour définir l'aspect général de l'écoulement et ses effets macroscopiques.

C'est ce qui se passe également, à un degré encore plus grand, en thermodynamique, et c'est la raison profonde de la notion de probabilité d'un état : sous un même état se cachent un grand nombre de situations microscopiques différentes.

Encore une fois, nous ne prétendons pas pour l'instant énumérer les grandeurs qui suffisent à définir l'état d'un écoulement. Nous prétendons simplement que leur nombre devient vite inférieur au nombre de degrés de liberté de cet écoulement. Il existerait donc une probabilité pour chaque état et l'état le plus souvent observé correspondrait donc bien au maximum de quelque chose : ce serait l'état de probabilité maximum.

Comme en thermodynamique, il est plus commode de considérer le logarithme de cette probabilité, afin d'assurer l'additivité pour deux régions indépendantes de l'écoulement. Voilà donc les deux propriétés principales que nous supposerons à cette quantité (nous la nommerons $Q$ ) : elle sera additive et représentera à une constante près (et au signe près comme nous le verrons plus loin) le logarithme d'une probabilité.

Dans tout ce qui suit, nous prendrons comme exemple le cas, souvent discuté dans la littérature, de la turbulence homogène et isotrope.

\section{Hiérarchie des échelles de longueur.}

Dans ce paragraphe, sans prétendre résoudre parfaitement le problème posé, nous voudrions souligner qu'il existe une différence entre ce qui résulte de l'existence de l'entropie en thermodynamique (équilibre thermique) et ce qui résulte de l'existence de $Q$ pour les écoulements turbulents.

Il est admis [5] qu'il existe en turbulence homogène et isotrope à trois dimensions une cascade d'énergie, des structures à grande échelle nourissant en énergie les échelles plus petites. L'idée physique souvent présentée est celle de grands tourbillons étirant de plus petits tourbillons et ainsi de suite.

Ces structures se distinguant par leur taille, il est d'usage d'utiliser un nombre d'onde comme label : $k$. Dans le schéma ci-dessus, il existe un certain arbitraire dans la façon dont la puissance $E$ venant des échelles supérieures est répartie entre les échelles de label donné $k$. Supposons que cet arbitraire soit levé par la recherche d'un extremum pour $Q$. Soient $\gamma_{\mathrm{i}}(e)$ la contribution à $Q$ d'une structure particulière $i$ et $\Gamma_{k, i}(E-e)$ la contribution des autres structures de même label $k$. Nous aurions donc à chercher l'extremum de

$$
\gamma_{\mathrm{i}}(e)+\Gamma_{k, i}(E-e)
$$

qui s'obtient pour :

$$
\frac{\partial \gamma_{i}}{\partial e}=\frac{\partial \Gamma_{k i}}{\partial E} \cong \frac{\partial \Gamma_{k}}{\partial E}
$$


où $\Gamma_{k}$ est la contribution totale des structures $k$. Par analogie avec la thermodynamique, $\Gamma_{k}$ ou $Q$ étant l'analogue de l'entropie et $E$ de l'énergie, $\frac{\partial \Gamma_{k}}{\partial E}$ serait l'inverse d'une « température dynamique ». On voit qu'elle se nivelle bien à l'intérieur d'une même échelle, mais pas entre échelles. Cette température dépendrait donc de $k$ au lieu d'être équirépartie comme on l'imaginerait par référence à la thermodynamique.

Signalons que des idées similaires à celles de ce paragraphe ont été présentées par T. M. Brown [3], qui le conduisent lui aussi à l'introduction d'une «température turbulente » dépendant de l'échelle. Partant de considérations plus générales que lui, sans référence directe à l'équation de Navier-Stokes, nous avons voulu rappeler que cette notion apparaissait aussi dans ce cadre.

\section{Formalisme.}

Nous développons à partir de ce paragraphe notre formalisme dans le cas de la turbulence homogène et isotrope. Suivant Landau [4], on peut considérer que, dans une situation homogène, les quantités pertinentes sont celles rapportées au volume unité. Nous supposerons donc que l'état turbulent le plus probable est celui qui minimise la quantité :

$$
\frac{Q}{V}=\int_{k_{1}}^{k_{2}} q\left(\varepsilon, \frac{\mathrm{d} \varepsilon}{\mathrm{d} k}, k\right) \mathrm{d} k
$$

sur tout intervalle $\left(k_{1}, k_{2}\right)$ ( $V$ est le volume).

Il convient de faire plusieurs remarques à ce niveau.

- On pourrait avancer des arguments qualitatifs justifiant la recherche d'un minimum plutôt qu'un maximum. Comme nous l'avons dit, nous n'accordons aucune valeur de rigueur à ces arguments et ne voulons pas sembler nous appuyer dessus. Disons simplement que, par la recherche d'un minimum, on obtient un formalisme plus agréable où $q$ est positif et tend vers 0 quand $k$ tend vers l'infini.

- $\varepsilon$, la puissance dissipée par unité de masse du fluide et $k$ sont les grandeurs traditionnellement supposées déterminer ce type d'état turbulent. Par la présence de $\frac{\mathrm{d} \varepsilon}{\mathrm{d} k}$, nous introduisons une liberté qui peut se justifier par l'intermittence de l'écoulement : il est clair par exemple que $\varepsilon$ moyen (qui se conserve dans la vision classique [5]) peut être différent de $\varepsilon$ le plus probable qui est celui dont nous parlons [6]. Les grandes fluctuations de $\varepsilon$ autorisent également que, par accident, il devienne très petit. Pour une telle petite valeur, $k$ deviendrait une échelle dissipative et cela ferait perdre en moyenne de l'énergie à la cascade.

Le problème variationnel posé par (1) est formellement l'analogue d'un problème de Mécanique où $q$ serait le Lagrangien, $\varepsilon$ la position et $k$ le temps. Par analogie, nous noterons $\dot{\varepsilon}=\frac{\mathrm{d} \varepsilon}{\mathrm{d} k}$. La solution est celle des équations de Euler-Lagrange :

$$
\frac{\mathrm{d}}{\mathrm{d} k}\left(\frac{\partial q}{\partial \dot{\varepsilon}}\right)=\frac{\partial q}{\partial \varepsilon} .
$$

La situation est la même qu'en Mécanique : nous ne pouvons pas aller plus loin sans expliciter q. Mais nous pouvons déjà remarquer que le résultat sera une équation différentielle du second ordre pour $\varepsilon(k)$. La solution sera déterminée par deux conditions aux limites correspondant aux grandes et aux petites échelles, par exemple la donnée des températures dynamiques en ces points. Ce formalisme introduit donc naturellement 
l'influence des grandes et des petites échelles sur l'écoulement moyen, via les conditions aux limites qu'elles imposent.

Dans les paragraphes suivants, nous introduirons successivement les hypothèses qui nous permettent de proposer une forme pour $q$. Nous tirerons les conséquences de chaque étape pour estimer le mieux possible la généralité des résultats obtenus.

\section{Quelques hypothèses raisonnables.}

4.1 FORME GÉNÉRALE DE $q$. - Une hypothèse dans la ligne de celle faite par Kolmogorov [5] pour déterminer le spectre d'énergie consiste à supposer que les conditions aux limites (grandes échelles et viscosité pour les petites échelles) ne figurent pas explicitement dans $q$. Avec les trois grandeurs :

$$
\varepsilon, \dot{\varepsilon}, k
$$

on ne peut faire qu'un nombre sans dimension :

$$
\eta=-\frac{k}{\varepsilon} \frac{\mathrm{d} \varepsilon}{\mathrm{d} k}=-\frac{k \dot{\varepsilon}}{\varepsilon}
$$

D'autre part, d'après notre définition (1) $q$ a la dimension de $k^{d-1}$ où $d$ est la dimension de l'espace (ici $d=3$ ). Donc

$$
q=k^{d-1} f(\eta) .
$$

Rappelons que cette forme de $q$ résulte de l'hypothèse de Landau ( $Q$ proportionnel à $V)$. Si $Q$ était proportionnel à une puissance non entière de $V: V^{\gamma}$, il faudrait remplacer $d$ par $3 \gamma$.

En reportant cette forme de $q$ dans l'équation de Euler-Lagrange (2), une algèbre simple permet de l'intégrer sous la forme :

$$
f^{\prime}(\eta)=\frac{\mathrm{d} f}{\mathrm{~d} \eta}=-A k^{-d}
$$

où $A$ est une constante. Nous retrouverons cette relation un peu plus loin.

4.2 Stabilité. - Le fait que $Q$ doive être minimum va nous donner une condition sur la convexité de $f$. Soit $\varepsilon_{\mathrm{m}}(k)$ la solution de (2) et $\eta_{\mathrm{m}}(k)$ la fonction $\eta(k)$ correspondante. Posons dans le cas général :

$$
\eta=\eta_{\mathrm{m}}+\delta \eta
$$

Comme $\frac{\eta}{k}=-\frac{\dot{\varepsilon}}{\varepsilon}=-\frac{\mathrm{d}}{\mathrm{d} k} \log \varepsilon$, on peut écrire, définissant par là la fonction $y$

$$
y(k)=+\log \frac{\varepsilon}{\varepsilon_{\mathrm{m}}}=-\int_{k_{1}}^{k} \frac{\delta \eta}{k^{\prime}} \mathrm{d} k^{\prime} .
$$

Dans la recherche du minimum de $Q$ les valeurs de $\varepsilon$ en $k_{1}$ et $k_{2}$ restent fixées. On a donc

$$
y\left(k_{2}\right)=-\int_{k_{1}}^{k_{2}} \frac{\delta \eta}{k^{\prime}} \mathrm{d} k^{\prime}=0 .
$$


En développant $Q$ en puissances de $\delta \eta$, on obtient :

$$
\frac{Q-Q_{0}}{V}=\int_{k_{1}}^{k_{2}} k^{d-1} f^{\prime}\left(\eta_{\mathrm{m}}\right) \delta \eta \mathrm{d} k+\frac{1}{2} \int_{k_{1}}^{k_{2}} k^{d-1} f^{\prime \prime}\left(\eta_{\mathrm{m}}\right) \delta \eta^{2} \mathrm{~d} k .
$$

La première intégrale dans le membre de droite est nulle. Cela résulte de la condition $y\left(k_{i}\right)=0$ ci-dessus et de la relation (5), c'est-à-dire, indirectement et comme il se doit, de la relation d'Euler-Lagrange. On peut même considérer cela comme une démonstration de la relation (5). La forme de la deuxième intégrale montre d'autre part que :

$$
f^{\prime \prime}(\eta)>0
$$

qui est la condition de convexité cherchée.

4.3 Distributions log-NORMALES. - C'est ici que nous allons nous servir de l'idée que $Q$ représente au signe près le logarithme d'une probabilité. D'après la forme de $Q-Q_{0}$, on voit que :

- Les $\delta \eta(k)$ sont des variables indépendantes et gaussiennes. En effet, si $\left\langle\delta \eta^{2}\right\rangle$ est proportionnel à $V^{-1},\left\langle\delta \eta^{3}\right\rangle$ sera proportionnel à $V^{-3 / 2}$ et le troisième terme du développement, proportionnel à $V^{-1 / 2}$, devient négligeable, ainsi que les suivants à la limite des grands volumes. Notons que le résultat serait le même si $Q$ était proportionnel à $V^{\gamma}$ avec $\gamma>0$.

- $y(k)$ (et donc $\log \varepsilon)$, combinaison linéaire de ces variables est gaussien. Le résultat est que $\varepsilon$ est log-normal.

Le fait que $\varepsilon$ soit log-normal correspond à une ancienne hypothèse de Kolmogorov et Obukhov [6]. Ils faisaient reposer cette hypothèse sur le fait que les fluctuations s'accumulaient de façon multiplicative (donc additive pour le logarithme) le long de la cascade d'énergie. Dans l'idée de Kolmogorov et Obukhov, la variance de cette distribution dépendait du vecteur d'onde comme $\log k$. Une telle variation correspond pour nous à un cas limite que nous examinerons à la fin de l'article.

Nous pouvons de façon générale calculer la variance de la distribution de $\log \varepsilon$. Pour présenter le calcul de façon simple, le mieux est d'approximer les intégrales par des sommes de Riemann :

$$
y=\sum_{i} \frac{\delta \eta_{i}}{k_{i}} \delta k=\sum_{i} u_{i}
$$

avec

$$
u_{i}=\frac{\delta \eta_{i}}{k_{i}} \delta k
$$

et $\delta k$ est le pas d'intégration

$$
Q-Q_{0}=\frac{V}{2} \sum_{j} k_{j}^{d-1} f^{\prime \prime}\left(\eta_{\mathrm{m}}\right)\left(\delta \eta_{j}\right)^{2} \delta k .
$$

Or, en utilisant (5) :

$$
f^{\prime \prime}\left(\eta_{\mathrm{m}}\right)=A d k^{-d-1} \frac{\mathrm{d} k}{\mathrm{~d} \eta}
$$


D'où

$$
Q-Q_{0}=\frac{a V d}{2} \sum_{j} \frac{\mathrm{d} k}{\mathrm{~d} \eta}\left(\frac{\delta \eta_{j}}{k_{j}}\right)^{2} \delta k=\frac{V A d}{2} \sum_{j} \frac{u_{j}^{2}}{\delta k \frac{\mathrm{d} \eta}{\mathrm{d} k}}
$$

La variance de $u_{j}$ est donc:

$$
\left\langle u_{j}^{2}\right\rangle=\frac{1}{V A d} \frac{\mathrm{d} \eta}{\mathrm{d} k} \delta k
$$

et la variance de $y$ est la somme des variances des $u_{i}$ :

$$
\left\langle y^{2}\right\rangle=\sum_{i}\left\langle u_{i}^{2}\right\rangle=\frac{1}{V A d} \sum_{i} \frac{\mathrm{d} \eta}{\mathrm{d} k} \delta k=\frac{1}{V A d} \int_{k_{1}}^{k} \frac{\mathrm{d} \eta}{\mathrm{d} k} \mathrm{~d} k .
$$

Dans un écoulement réel, la variance de $\log \varepsilon$ est nulle du côté des grandes échelles où l'on impose la puissance ; là également $\eta$ est très petit. On peut donc écrire :

$$
\left\langle\log ^{2} \frac{\varepsilon}{\varepsilon_{\mathrm{m}}}\right\rangle=\frac{1}{V A d} \eta \text {. }
$$

Nous insistons sur le fait que ce résultat et ceux qui précèdent sont indépendants des hypothèses qui vont être faites dans les paragraphes qui suivent.

\section{Loi de puissance et géométrie de l'écoulement.}

La condition de convexité (7) impose peu de contraintes sur $f$. Pour aller plus loin dans notre analyse, nous supposerons une loi de puissance pour $f^{\prime}$ :

$$
f^{\prime}(\eta)=-C \eta^{-\alpha}
$$

A ce niveau, cette loi peut paraître arbitraire. Sa signification intuitive viendra de l'interprétation physique que nous ferons de $\eta$ d'une part, et d'autre part de l'interprétation géométrique des lois que nous obtiendrons.

$\mathrm{Au}$ voisinage des échelles dissipatives, $\eta$, étant proportionnel à $\dot{\varepsilon}$, est proportionnel à la viscosité à écoulement constant. Au moins dans ce voisinage, il se présente donc comme l'inverse d'un nombre de Reynolds local. En tant que tel, on s'attend à ce qu'il augmente quand $k$ augmente, le nombre de Reynolds devenant inférieur à 1 dans les échelles purement dissipatives. Donc $\frac{\mathrm{d} \eta}{\mathrm{d} k}$ devrait être positif. En utilisant la condition (7) et l'équation (5), on voit alors que $A, C$ et $\alpha$ doivent être positifs. En rapprochant (5) et (10), on voit d'autre part que :

$$
\eta=\left(\frac{C}{A}\right)^{1 / \alpha} k^{d / \alpha}
$$

d'où

$$
\frac{\dot{\varepsilon}}{\varepsilon}=-\left(\frac{C}{A}\right)^{1 / \alpha} k^{\beta-1}
$$

en posant $\beta=\frac{d}{\alpha}$. En intégrant :

$$
\varepsilon=\varepsilon_{0} \exp \left[-\left(\frac{k}{k_{0}}\right)^{\beta}\right]
$$


où $\varepsilon_{0}$ et $k_{0}$ représentent les deux constantes d'intégration que nous mentionnons à la fin du paragraphe $3: \varepsilon_{0}$ correspond à la puissance injectée aux grandes échelles et $k_{0}$, vecteur d'onde de coupure du spectre correspond aux petites échelles.

Venons-en à présent à l'interprétation géométrique. De la relation :

$$
\frac{\mathrm{d}}{\mathrm{d} k} f(\eta)=f^{\prime}(\eta) \frac{\mathrm{d} \eta}{\mathrm{d} k}
$$

et de (5), (11), on déduit que $f$ est proportionnel à $k^{-d+\beta}$ ( $f$ tend vers 0 quand $k$ tend vers l'infini). Il en résulte que :

$$
q(k) \propto k^{\beta-1} .
$$

Dans un espace de dimension $d$, on s'attend à une dépendance en $k^{d-1}$ pour avoir un nombre de degrés de liberté $(Q)$ variant comme $k^{d}$. Donc $\beta$ se présente comme la dimension effective de l'écoulement.

En utilisant un exemple simple où $\beta$ serait égal à 1 , on peut d'autre part voir que cette dimension est la co-dimension du lieu de dissipation maximale. Imaginons en effet que cette dissipation maximale ait lieu sur une surface de dimension 2. La seule direction dans laquelle la vitesse varie notablement est la normale à cette surface : le champ de vitesse est alors virtuellement de dimension 1.

Cela donne de l'importance à la relation (11). Certes, le flux d'énergie $\varepsilon$ en fonction du nombre d'onde $k$ n'est pas une quantité facile à mesurer. Mais une telle dépendance exponentielle doit se retrouver dans d'autres quantités, comme le spectre d'énergie $E(k)$. D'autre part, il devrait être possible de mesurer la dimension fractale $d^{\prime}$ du lieu de dissipation maximale. Il pourrait suffire d'observer les maxima de la dérivée de la vitesse en un point et de considérer cette succession de points dans le temps comme la coupe de ce lieu de dissipation par une ligne de dimension 1. La dimension de cet ensemble de points serait alors [7] :

$$
1+d^{\prime}-d=1-\beta
$$

On peut donc vérifier expérimentalement la concordance avec l'exposant $\beta$ tiré du spectre $E(k)$.

Avant de clore ce paragraphe, il convient de signaler que de nombreux auteurs, impossibles à citer tous, ont prédit un spectre en $\exp \left[-\left(\frac{k}{k_{0}}\right)^{\beta}\right]$. A notre connaissance, aucune de ces prédictions n'était liée à la minimisation d'une quantité. Dans aucun de ces travaux il n'est explicitement signalé que l'exposant $\beta$ doit dépendre du nombre de Reynolds comme nous le montrerons au paragraphe suivant. Cependant, l'hypothèse d'une dimension fractale pour la structure dissipative a depuis longtemps été faite $[8,9]$ et donc l'importance de la géométrie de l'écoulement sur ses propriétés statistiques a déjà été reconnue. A ce sujet, il convient de signaler une divergence de point de vue entre cet article et les précédents. Considérant que les vecteurs d'onde les plus importants sont perpendiculaires à la structure dissipative, nous négligeons complètement les variations le long de cette structure ; nous en déduisons donc un nombre de degrés de liberté variant comme $k^{\beta}$ pour les vecteurs d'ondes inférieurs à $k$. Dans l'interprétation classique au contraire, cette direction perpendiculaire est considérée comme " morte » et les seules variations prises en compte sont le long de la structure dissipative. Le nombre de degrés de liberté est alors proportionnel à $k^{3-\beta}$ [10]. Ces deux interprétations reposent manifestement sur des visions différentes de l'écoulement. En particulier, dans l'interprétation classique $\beta$ est fixe, indépendant du nombre de Reynolds. Cette divergence 
de point de vue mériterait une plus longue discussion, que nous ne pouvons faire dans cet article.

Une mention doit également être faite de l'article de U. Frisch et R. Morf [11]. Ces auteurs expliquent les intermittences observées dans l'écoulement par la présence de singularités de la vitesse dans le plan complexe des variables d'espace. Cela doit conduire pour chacune de ces intermittences à un spectre en $\exp \left[-\left(\frac{k}{k_{0}}\right)\right]$ qui peut donner $\exp \left[-\left(\frac{k}{k_{0}}\right)^{\beta}\right]$ avec $\beta<1$ pour certaines répartitions des singularités. Ces hypothèses ont reçu une certaine confirmation expérimentale [12]. Bien que ne partant pas des mêmes bases, cette description est donc assez proche de ce que nous avons exposé, la partie réelle de la position des singularités donnant le lieu de dissipation maximale. Dans le même esprit, et bien qu'il n'y soit encore pas question de minimisation, il faut citer les expériences du groupe de l'Université de Pittsburg [13] qui sont interprétées comme montrant une dépendance de la géométrie de l'écoulement avec le nombre de Reynolds.

\section{Ordres de grandeur et lois limites.}

Dans ce paragraphe, nous répondrons d'abord à un souci : pour que la loi en exp $\left[-\left(\frac{k}{k_{0}}\right)^{\beta}\right]$ ait un sens, il faut qu'elle soit valable pour $\frac{k}{k_{0}}$ suffisamment grand. Sinon, elle peut ne représenter que le début du développement d'une loi tout à fait différente. Or il existe une coupure naturelle en $k$ pour ce formalisme : il n'a pas de sens pour les échelles fortement dissipatives où le nombre de degrés de liberté est nul. La coupure est donc $\frac{1}{\delta}$ où $\delta$ est «l'épaisseur » de la surface de dissipation maximale. La façon dont nous évaluerons $\delta$ nous permettra de déduire la loi limite de variation de $\beta$ pour les très grands Reynolds. Enfin, dans cette même limite, nous retrouverons que la variance de la distribution de $\log \varepsilon$ est en $\log k$, qui est la loi postulée par Kolmogorov et Obukhov [6].

Pour estimer $\delta$, remarquons d'abord que si $d^{\prime}$ est la dimension fractale de la surface de dissipation maximale, le volume où a lieu la dissipation est de l'ordre de :

$$
L^{d^{\prime}} \delta^{d-d^{\prime}}=L^{d-\beta} \delta^{\beta}
$$

où $L$ représente la grande échelle dans l'écoulement.

On peut donc écrire :

$$
\varepsilon L^{d}=\nu \frac{v_{\delta}^{2}}{\delta^{2}} L^{d-\beta} \delta^{\beta}
$$

où $v_{\delta}$ est la vitesse caractéristique à l'échelle $\delta, \nu$ la viscosité cinématique et

$$
\varepsilon=\varepsilon\left(\delta^{-1}\right)=\varepsilon_{0} \exp \left[-\left(k_{0} \delta\right)^{-\beta}\right]
$$

est l'énergie dissipée par unité de masse à cette échelle. Cela n'est pas toute l'énergie injectée au niveau de la grande échelle car beaucoup d'énergie a été dissipée dans les échelles intermédiaires. Cependant, dans ces échelles intermédiaires, la dissipation était accidentelle et loin de représenter toute l'énergie venant de l'échelle immédiatement supérieure. D'autre part :

$$
\varepsilon=\frac{v_{\delta}^{3}}{\delta}
$$

ce qui signifie que le temps d'amortissement de l'énergie est $\delta / v_{\delta}$. 
En combinant (12) et (14) :

$$
v_{\delta}=\nu L^{-\beta} \delta^{\beta-1}
$$

Les équations (13), (14) et (15) donnent alors :

$$
\exp \left[-\left(k_{0} \delta\right)^{-\beta}\right]=\frac{\nu^{3}}{\varepsilon_{0}} L^{-3 \beta} \delta^{3 \beta-4}=\left(\frac{l_{K}}{\delta}\right)^{4-3 \beta}\left(\frac{l_{K}}{L}\right)^{3 \beta}
$$

où $l_{K}=\left(\frac{\nu^{3}}{\varepsilon_{0}}\right)^{1 / 4}$ est la valeur classique de la longueur de Kolmogorov [4, 5].

Considérons d'abord un cas où $\beta$ serait de l'ordre de 1 . Il est clair que $l_{K}$ est beaucoup plus proche de $\delta$ que de $L$. Donc :

$$
\exp \left[-\left(k_{0} \delta\right)^{-\beta}\right] \cong\left(\frac{l_{K}}{L}\right)^{3 \beta} \cong R_{\mathrm{e}}^{-3 \beta / 4}
$$

$R_{\mathrm{e}}^{3 \beta / 4}$ est un nombre très grand si $\beta$ est de l'ordre de 1 . Cela signifie que la loi exponentielle est valable sur toute la partie observable du spectre.

Tournons-nous à présent vers la limite $R_{\mathrm{e}} \rightarrow \infty$. Dans cette limite, comme on ne peut faire qu'une longueur avec $\varepsilon_{0}$ et $\nu, k_{0} \delta$ et $\left(l_{K} / \delta\right)$ doivent tendre vers des limites finies. La relation (16) impose alors que $\left(l_{K} / L\right)^{\beta}$ tende vers une limite. En prenant le logarithme de cette quantité et en se souvenant que $l_{K} / L=R_{\mathrm{e}}^{-3 / 4}$, cela signifie que :

$$
\beta \log R_{\mathrm{e}} \rightarrow \beta_{0} .
$$

Il est logique intuitivement que $\beta$ tende vers 0 . Cela signifie que la surface de dissipation maximale se «froisse » de plus en plus, acquérant une dimension de plus en plus proche de 3 [13]. La relation (17) nous dit la façon dont se fait cette approche à 0 .

Si $\beta \rightarrow 0$, on peut écrire :

$$
\left(\frac{k}{k_{0}}\right)^{\beta}=\exp \left[\beta \log \left(\frac{k}{k_{0}}\right)\right] \cong 1+\beta \log \frac{k}{k_{0}}=\beta \log \frac{k}{k_{1}}
$$

où

$$
k_{1}=k_{0} \exp -\frac{1}{\beta} \cong k_{0} R_{\mathrm{e}}^{-1 / \beta_{0}} .
$$

Comme $\eta=-\frac{\dot{\varepsilon} k}{\varepsilon}=\beta\left(\frac{k}{k_{0}}\right)^{\beta}$, on retrouve bien, par l'équation (9) une variance en $\log k$ pour $\log \varepsilon$ :

$$
\left\langle\log ^{2} \frac{\varepsilon}{\varepsilon_{\mathrm{m}}}\right\rangle=\frac{1}{V A d} \eta=\frac{\beta_{0}^{2} \log \left(k / k_{1}\right)}{V A d\left(\log R_{\mathrm{e}}\right)^{2}}
$$

\section{Conclusion.}

Rappelons tout d'abord ici l'originalité de notre démarche. Dans tous les essais antérieurs concernant un principe d'extremum, la quantité en question était directement reliée aux caractéristiques de l'écoulement. Ce fut par exemple dans de nombreux cas la dissipation totale. Ici, nous partons du principe qu'il doit en exister une, sans l'expliciter. Nous déduisons sa forme de considérations de symmétrie. 
Notre but principal dans cet article était de montrer que l'existence d'une quantité à minimiser n'était pas par principe contraire à ce que l'on connaît des écoulements turbulents (alors que l'on sait par exemple que la dissipation totale n'est pas toujours minimale). Nous nous sommes donc attachés à retrouver des résultats qui, s'ils ne sont pas toujours démontrés, sont du moins admis et pourraient pour certains ne pas nécessiter de justification : distribution $\log$-normale pour $\varepsilon$, loi en «exponentielle étirée " pour le spectre, variance en $\log k$ pour Log $\varepsilon$ à la limite des grands Reynolds. Nous avons également obtenu des résultats que nous pensons originaux comme la loi de variation de l'exposant $\beta$. L'utilité de ce formalisme nous semble donc acquise.

\section{Remerciements.}

Ce travail doit beaucoup à l'enthousiasme de Giovanni Zocchi qui a ravivé ma foi en ce type d'approche. R. Rammal m'a beaucoup aidé par ses remarques lumineuses. Les discussions avec S. Thomae et M. Papoular m'ont également permis d'y voir plus clair. Je tiens enfin à remercier le rapporteur pour ses critiques constructives et les références qu'il m'a signalées.

\section{Bibliographie}

[1] Von Karman, T., Collected Works (Butterworth's Scientific Publications, London 1956) Vol. 2 p. 1914, cité par BETCHOV, R., The Physics of Fluids 7 (1964) 1160.

[2] Benzi, R., Vitaletti, M. et Vulpiani, A., J. Phys. A 15 (1982) 883.

[3] Brown, T. M., J. Phys. A 15 (1982) 2285.

[4] LANDAU, L., Lifchitz, E., Mécanique des Fluides (Ed. Mir).

[5] Kolmogorov, A. N., Dok. Akad. Nauk. (SSSR) 30 (1941) 299. Voir aussi la référence [4].

[6] Kolmogorov, A. N., Mécanique de la Turbulence (Colloque International du CNRS à Marseille) Ed. CNRS Paris (1962) p. 447 et J. F. M. 13 (1962) 82. Voir aussi Obukhov, A. M., J. F. M. 13 (1962) 77 et YAglom, A. M., Dok. Akad. Nauk. SSSR 166 (1966) 49.

[7] Rammal, R., Communication privée. Cela vient de ce que la co-dimension de l'intersection est la somme des co-dimensions. Pour des dimensions entières, ce résultat est évident, la codimension étant aussi le nombre d'équations qui définissent la variété.

[8] Mandelbrot, B., Lectutres Notes in Mathematics, ed. R. Temam (Springer) 565 (1976) 121.

[9] Firsch, U., Sulem, P. L., Nelkin, M., J. F. M. 87 (1978) 719.

[10] Kraichnan, R. H., Phys. Fluids 28 (1985) 10 et Paladin, G. et Vulpiani, A., Phys. Rep. 156 (1987) 147.

[11] Frisch, U., Morf, R., Phys. Rev. A 23 (1981) 2672.

[12] Dombre, T., Gagne, Y. et Hopfinger, E. J., Advances in Turbulence (G. Comte Bellot et J. Mathieu, ed. Springer Verlag, 1986) p. 204.

[13] Tong, P., Goldburg, W. I., Chan, C. K. et Sirivat, A., Phys. Rev. A 37 (1988) 2125. 